\title{
An Energy-Dispersive X-Ray Fluorescence Spectrometry and Monte Carlo simulation study of Iron-Age Nuragic small bronzes ("Navicelle") from Sardinia, Italy is
}

\author{
Nick Schiavon ${ }^{\mathrm{a}, *}$, Anna de Palmas ${ }^{\mathrm{b}}$, Claudio Bulla ${ }^{\mathrm{b}}$, Giampaolo Piga ${ }^{\mathrm{c}}$, Antonio Brunetti ${ }^{\mathrm{c}, \mathrm{d}}$ \\ a HERCULES Laboratory for the Study and Conservation of Cultural Heritage, University of Évora, Portugal \\ b Dipartimento di Storia, Scienze dell'Uomo e della Formazione, Università di Sassari, Italy \\ c Dipartimento di Scienze Politiche, Scienze della Comunicazione, Ingegneria dell'Informazione, Università di Sassari, Italy \\ d CIRTEBEC, Università di Sassari e Cagliari, Italy
}

\section{A R T I C L E I N F O}

Article history:

Received 15 November 2015

22 July 2016

Accepted 25 July 2016

Available online 26 July 2016

\section{Keywords:}

Energy Dispersive X-Ray Fluorescence Spec-

troscopy

Monte Carlo simulations

Nuragic bronzes

Navicelle

Sardinia

\begin{abstract}
A B S T R A C T
A spectrometric protocol combining Energy Dispersive X-Ray Fluorescence Spectrometry with Monte Carlo simulations of experimental spectra using the XRMC code package has been applied for the first time to characterize the elemental composition of a series of famous Iron Age small scale archaeological bronze replicas of ships (known as the "Navicelle") from the Nuragic civilization in Sardinia, Italy. The proposed protocol is a useful, nondestructive and fast analytical tool for Cultural Heritage sample. In Monte Carlo simulations, each sample was modeled as a multilayered object composed by two or three layers depending on the sample: when all present, the three layers are the original bronze substrate, the surface corrosion patina and an outermost protective layer (Paraloid) applied during past restorations. Monte Carlo simulations were able to account for the presence of the patina/corrosion layer as well as the presence of the Paraloid protective layer. It also accounted for the roughness effect commonly found at the surface of corroded metal archaeological artifacts. In this respect, the Monte Carlo simulation approach adopted here was, to the best of our knowledge, unique and enabled to determine the bronze alloy composition together with the thickness of the surface layers without the need for previously removing the surface patinas, a process potentially threatening preservation of precious archaeological/artistic artifacts for future generations.
\end{abstract}

(c) 2016 Published by Elsevier B.V.

\section{Introduction}

Energy Dispersive X-Ray Fluorescence Spectrometry (EDXRF) is a non-destructive analytical technique with wide applications in several research areas including the Cultural Heritage/Conservation Science one [1-17]. Performing quantitative EDXRF analyses on archaeological and Cultural Heritage metallic artifacts is, however, particularly challenging due to their complex multi-layered internal structure. "Layers" may include: a) the original metal and/or alloy substrate (gold, silver, bronze etc.); b) surface patinas enriched in corrosion products (sulphides, oxides and/or chlorides compounds) due to attack by agents present both in the atmospheric and/or burial environments; c) protective treatments applied in past conservation interventions; d) soil derived incrustations from the archaeological burial environment [10-14]. In cases where the layered structure is not homogeneous, the separation between two or more adjacent layers is not well defined

\footnotetext{
th Selected Paper from the Colloquium Spectroscopicum Internationale XXXIX (CSI 2015)

Figueira da Foz, Portugal, 30 August - 3 September 2015.

* Corresponding author.

E-mail address: schiavon@uevora.pt (N. Schiavon).
}

and/or when the layers are very thin, meaningful results on the bulk metal composition are even more difficult to obtain. In order to estimate layers thickness and composition in such multi-layered metal objects, a method based on estimating changes in the theoretical ratios of selected fluorescence line intensities due to the attenuation of the layers crossed by the radiation has been recently proposed $[4,7,18,19]$. However, this method strongly depends on the specific XRF setup used. In the aforementioned studies, for instance, a monochromatic X-Ray beam obtained by placing a filter at the output of the X-Ray tube has often been used: this setup, though, affects the quality of the results. Moreover, in some of these studies, the metal objects were subjected to a pre-analysis cleaning treatment to remove any surface corrosion patina and/or of protective layer present, a practice not always feasible when dealing with precious and unique artistic and/or archaeological objects. The methodological approach proposed in the current study involving Monte Carlo simulation tests does not require any constraint on the setup or on the XRF excitation spectrum nor it requires pre-treatment by potentially damaging cleaning procedures $[4,7]$.

In this study, the EDXRF/Monte Carlo Simulation protocol has been tested on a series of Iron Age (900-600 BCE) small-scale bronze replicas of ships, called "Navicelle" from the Nuragic civilization in Sardinia, Italy. 
Other examples of the application of Monte Carlo simulation to XRF analyses can be found in the literature [20-25], but the Monte Carlo used here shows some innovative features allowing the production of a high quality spectrum in just a few minutes (see Materials and methods section).

From an archaeological point of view, these bronzes has been usually associated with sacred sites this testifying their close, religious links with the sea which was a typical trait of the Nuragic culture in the island [26]. The "Navicelle" were probably used as lucernas (small lamps) in ex-votos offerings. Only a total of 150 Nuragic authentic "Navicelle" artifacts have been so far discovered in Sardinia but, due to their high artistic as well as archaeological significance, the commerce of these unique artifacts in local antiques fairs has been thriving for quite a long time thus stimulating the growth of a well-developed forgery industry. Despite their significance, no systematic material study has been ever carried out to shed light on their raw material provenance, production technology and objects authenticity. Aim of this work was therefore two-folded: a) to test the applicability of combining EDXRF data with Monte Carlo simulations in determining the bronzes bulk composition without the need for removing the alloy's bronze corrosion and/or protective patina; b) to provide compositional data on the unique Nuragic artifacts by nondestructive EDXRF spectrometry.

\section{Materials and methods}

Three "Navicelle" were selected for investigation: 2 boats (boat $n$. 1347 from an unknown locality in Sardinia and the so-called and famous "Re Sole" boat from the Badde Rupida Nuragic monument in Padria (Sassari), Fig. 1a and b) which are stored at the G.A. Sanna Museum in Sassari and 1 boat (boat n. 36/41 from Bultei, Northern Sardinia) which is stored at the National Archaeological Museum of Cagliari, Italy.

EDXRF analyses were performed using a portable X-Ray instrumentation formed by a SDD detector coupled with a DSP Multichannel analyzer (1-2-3 system manufactured by Amptek) and an Ag-anode X-Ray tube (mini-X manufactured by Amptek). Both detector and X-Ray tube are freely positionable, allowing to select the best geometrical setup for the analysis. However, the more common setup is formed by the detector placed vertically, $2-3 \mathrm{~cm}$ far, from the sample surface with the X-Ray tube forming an angle with the detector of about $30^{\circ}$, still $2-3 \mathrm{~cm}$ far from the sample surface. This position is preferable to the more standard $45^{\circ}-45^{\circ}$ configuration, because it allows to minimize the effects due to surface roughness. No collimation was used on the detector, while the X-Ray tube used a $1 \mathrm{~mm}$ wide collimator. The spot size was about $2 \mathrm{~mm}$. Each measurement lasted about $4 \mathrm{~min}$. A Monte Carlo model of the sample analyzed was then built assuming the two and/or three multilayered structure previously mentioned, although other type of structures were also tested. Our approach was based on the use of a Monte Carlo simulation (MC) following a probabilistic simulation of the X-Ray interaction with matter. Usually several millions of photon interactions need to be simulated before a good simulation of real experiments can be produced. The general aim of MC codes in the context here examined is to simulate a wide variety of X-Ray experimental set-ups: this lack of specialization, however, has the drawback that, in order to achieve an adequate simulation of a XRF analysis, a considerable amount of simulation time, (in the order of several hours or days) is usually required. To overcome this problem, several specialized MC codes have been developed. These codes are able to perform simulations only for a reduced set of X-Ray experiments, i.e. at energies $<100 \mathrm{keV}$. This limitation, however, does not introduce a true restraint on the XRF experiments that can be performed, which are typically run at energies around $40-50 \mathrm{keV}$ in most applications, particularly in the Cultural Heritage/Conservation Science sector. Using the aforementioned specialized codes, the speed of the simulation run can be dramatically increased: in fact, a XRF analysis can be adequately simulated in just a couple of minutes. This time is comparable to the experimental acquisition time and therefore the simulation can be a)

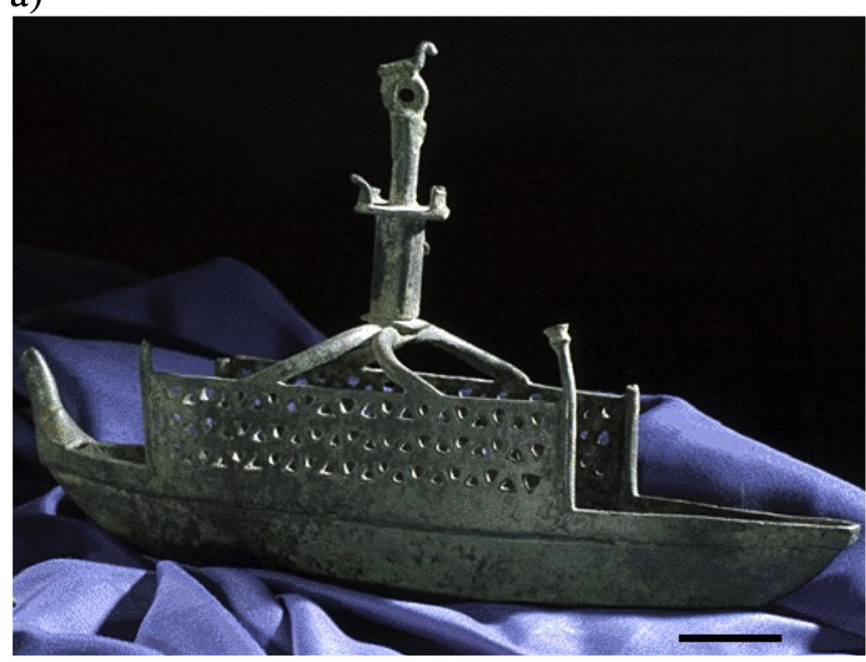

b)
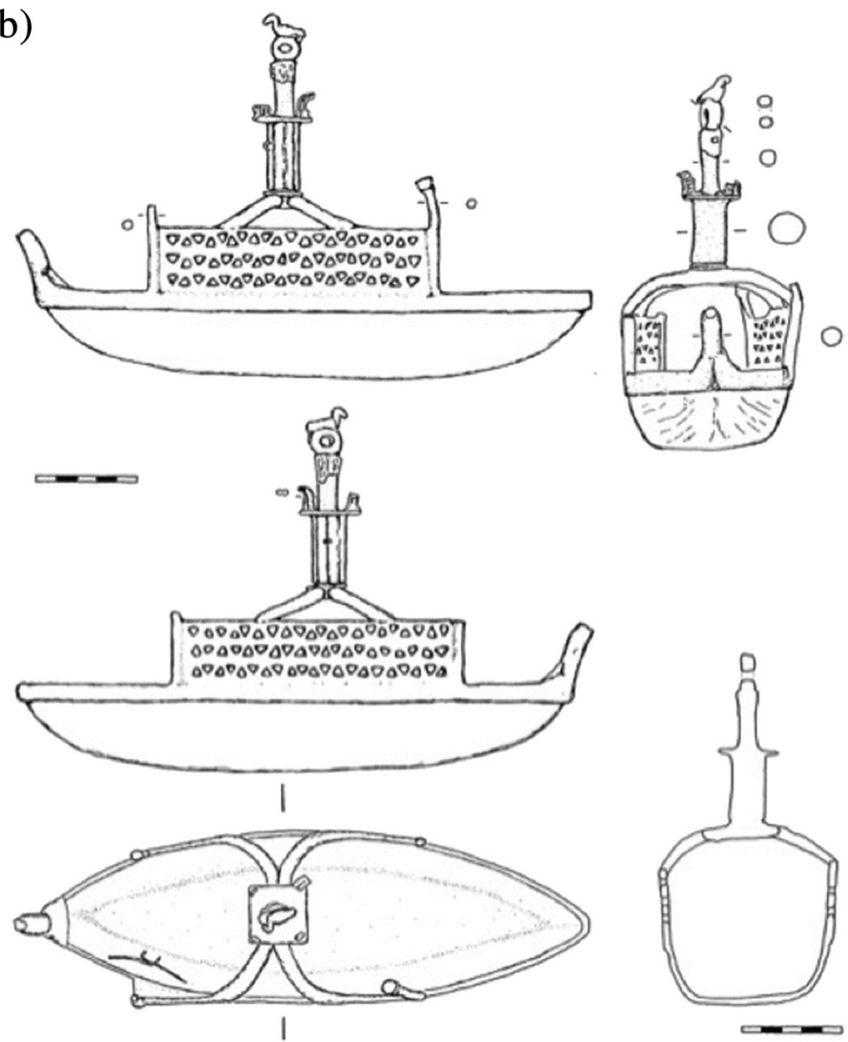

Fig. 1. Iron-Age bronzes from Sardinia, Italy: a) "Navicella" Re Sole, Padria, Sassari, scale bar $=4 \mathrm{~cm}$; b) "Navicella" Re Sole, Padria, Sassari, archaeological drawing, scale bars $=6 \mathrm{~cm}$.

regarded as being performed in real time. Two of such fast and specialized MC codes are readily available [27-32]. The first one was developed specifically for XRF experiments, while the second one can be also used to simulate radiographic, $\mathrm{CT}$ and phase contrast simulations. Both codes are based on the Xraylib database [33,34]. In the current study, we used the second one, called XRMC [31]. Before running the simulation, MC codes require a detailed description of both sample's composition and structure. From the point of view of the authors, one of the most critical parameter in this respect is the X-Ray spectrum emitted by the source. Here we have used the real spectrum emitted from the source, corrected for attenuation effects due to ambient air as well as to the presence of the detector windows. Its correctness has been tested on several reference samples. The quality of the simulation is assessed by comparing, 
at first visually and then by performing a chi-squared test, simulated and measured spectra: when the simulated spectrum matches exactly the experimental one, the sample can be determined both in its composition and structure. The match must be as much accurate as possible, because there could be several sets of compositions and structures able to produce a spectrum similar to the experimental one and in this sense several different models must be tested. This is mandatory particularly in the case of multilayered structures, such as the corroded metal objects under investigation, where an element can be present in more than one layer at the same time.

\section{Results and discussion}

As already mentioned, in textural terms, a typical bronze artifact, such as the ones described here, can be considered as a multi-layered object formed by a superficial corrosion layer superimposed to a bronze layer representing the substrate. Additionally, a protective Paraloid-like protective layer applied on the surface during past restoration interventions can often be found. The presence of these surface patinas makes it often harder to obtain a good estimate for the model. These may happen for two reasons: a) either the superficial patina is too thick to allow $\mathrm{X}$-Ray radiation emitted from the bronze substrate to reach the detector or b) the patina itself increases the roughness/irregularity of the artifact in such a way to make modeling almost impossible. In the first case, modeling the bronze object is impossible while in the second case surface roughness can and must be considered. A rough surface may introduce incontrollable changes on the measured spectra, such as energy dependent changes in peak intensities and/or a modulation of the background. However, the effect due to surface roughness can be reduced in three ways, namely: a) by choosing the geometry and the size of the beam; b) by introducing in the code a mathematical reproduction of the surface where some of parameters can by changed at runtime or: c) by introducing a reproduction of the real surface at the zone where the measurement was performed. At present, only the first two approaches can be exploited, while the last one is currently under development due to the difficulties to translate a 2D picture of the surface into a tridimensional model.

The EDXRF spectra, measured and simulated, of sample n. 1347 are depicted in Fig. 2. Its structure can be considered as a three layered one, where the first layer is a $150 \mu \mathrm{m}$ thick Paraloid varnish, the second one is a $1 \mu \mathrm{m}$ thick corrosion layer and finally the substrate "layer" is a typical ternary alloy ( $\mathrm{Cu} 79 \%-\mathrm{Pb} 10 \%-\mathrm{Sn} 10 \%$ ) with other minor elements $(<0.5 \%)$.

The Re Sole boat is the most important and, from a structural point of view, complex boat. For this reason, it has been analyzed at different

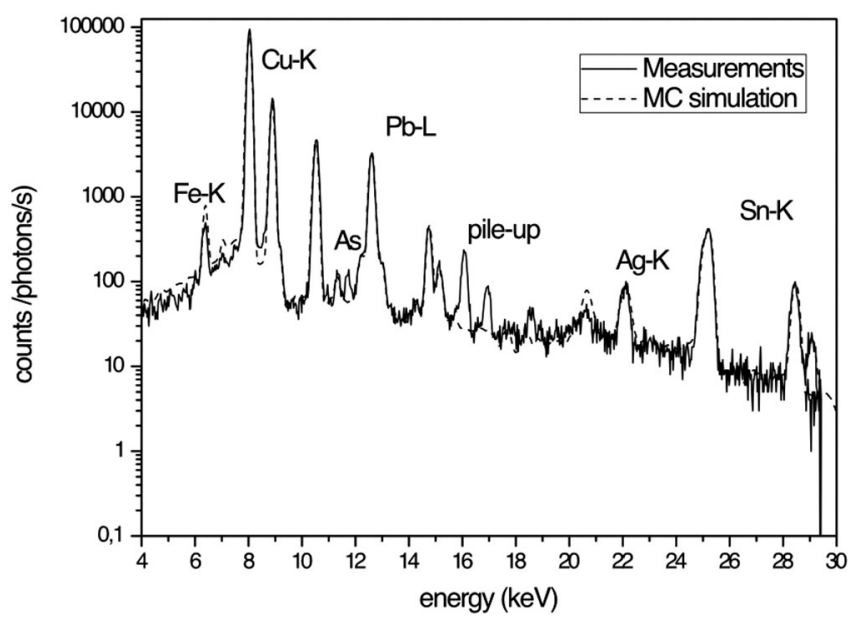

Fig. 2. EDXRF and Monte Carlo simulation spectra of Iron-Age "Navicelle" bronzes from Sardinia: 1347 boat, analysis on the main body.

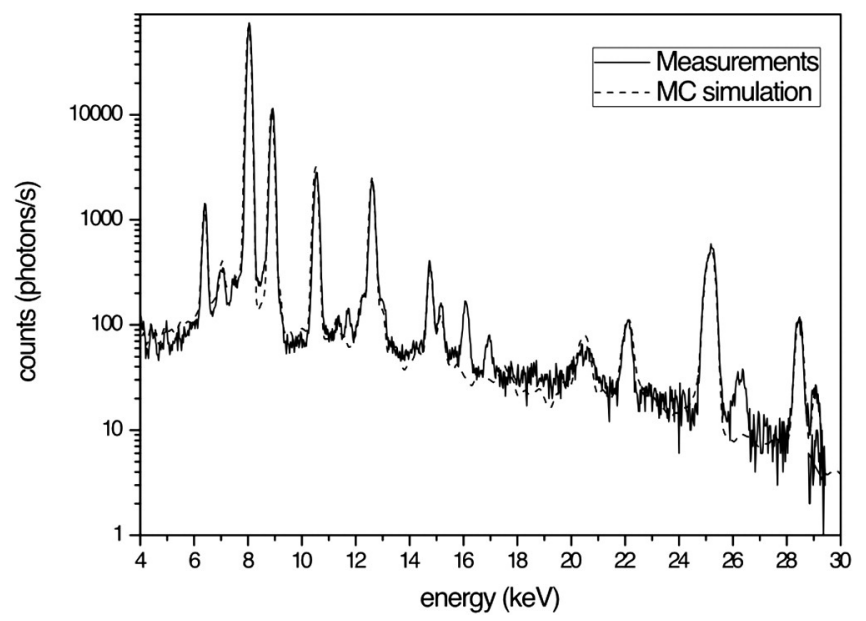

Fig. 3. EDXRF and Monte Carlo simulation spectra of Iron-Age "Navicelle" bronzes from Sardinia: Re Sole boat, analysis on left side of the hull.

spots in order to detect possible variations in the alloy's composition: the main body of the boat (analysis on the left side of the hull), the main mast base and the base of the station at the top of the main mast, the small mast and the ring. In all the analyzed areas, the bronze substrate appears to be a ternary alloy but the relative elemental percentages display some significant variations. The boat main body is made up by a Paraloid surface layer $200 \mu \mathrm{m}$ thick and a bronze substrate showing $87 \% \mathrm{Cu}, 3 \% \mathrm{Sn}$ and $10 \% \mathrm{~Pb}$ (Fig. 3). The station base is formed by 81.8\% Cu, 1\% Fe, 0.6\% Ag, 9.0\% Sn and 8.0\% Pb (Fig. S1, Appendix). The small mast has a similar composition while the ring shows higher Fe contents (5\% - Figs. S2 and S3, Appendix).

The Bultei boat sample is also covered by a layer of Paraloid varnish (30-140 $\mu \mathrm{m}$ thick) and by about $3 \mu \mathrm{m}$ of corrosion patina. The bronze alloy is again a ternary one showing compositional variations between the internal and the external side of the boat. In the internal side the presence of a thick patina does not allow to determine exactly its composition although it appears to contain the same elements of the external one, as it would be expected. On the external side, the composition is $79 \% \mathrm{Cu}, 3 \% \mathrm{Sn}$ and $15 \% \mathrm{~Pb}$, with small amount of $\mathrm{Ag}$ and $\mathrm{Sb}$. About $1 \%$ of $\mathrm{Zn}$ is also observed (Fig. 4).

Chemical and metallographic analyses performed in the past on Nuragic ingots and artifacts highlighted compositional variations with some displaying high $\mathrm{Cu}$ while others high $\mathrm{Pb}$ and $\mathrm{Fe}$ contents. For instance, a study on Nuragic "Navicelle" objects stored in private collections across Europe [35] revealed lower $\mathrm{Sn}$ and $\mathrm{Pb}(<2 \%)$ contents.

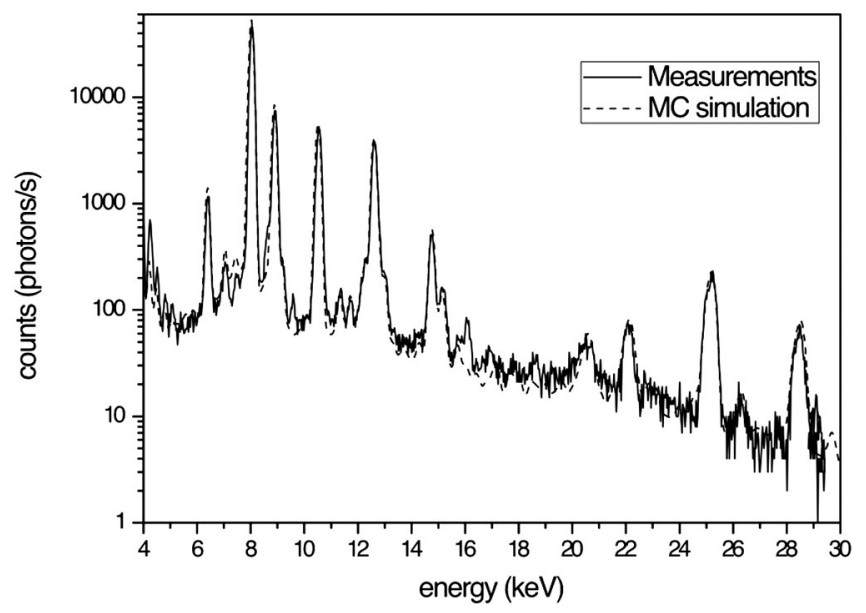

Fig. 4. EDXRF and Monte Carlo simulation spectra of Iron-Age "Navicelle" bronzes from Sardinia: Bultei boat, analysis on the external side of the main body. 
The latter objects, though, being part of private collections are most likely to include forged materials and, therefore, are often of uncertain provenance and these compositional results should be treated with caution. Another analysis, albeit performed only on a single fragment from a site near the city of Oliena in Sardinia [36], revealed low concentrations of $\mathrm{Sn}(0,37 \%)$ while displaying $5,5 \%$ of $\mathrm{Pb}$.

\section{Conclusions}

The use of Monte Carlo simulations on XRF experimental spectra permitted to obtain an excellent reproduction of the measured spectrum with a good description of the sample analyzed both from the point of view of its composition and structure. Samples were modeled as flat multilayered objects formed by two (in the Re Sole boat sample) or three (in samples 1347 and Bultei) layers: in the two layered model case, the surface corrosion layer was modeled as being 150-400 $\mu \mathrm{m}$ thick with the substrate layer considered of infinite thickness from the point of view of X-Ray absorption. The thin surface layer was formed by a Paraloid-like varnish used as protective agent in past conservation interventions. This layer does not contribute with characteristic peaks to the XRF spectrum and so its composition and thickness estimate are essentially based on the comparison between the simulated background with the experimental one, i.e. a thinner layer will produce a lower background while a thicker one will produce a higher background. The thickness is adjusted until the two backgrounds become perfectly superimposed to each other. The bronze composition varies depending on the artifact and/or specific area analyzed. For example, the ring section of the Re sole sample (Figs. 1 and 3) shows a larger concentration of Fe with respect to the other sections. Notwitstanding these variations, all samples examined were found to be made up by $\mathrm{Cu}, \mathrm{Pb}$ and $\mathrm{Sn}$ with minor Fe and Ag. The average elemental composition in wt\% for the Sassari/Cagliari samples is as follows: Cu 78.5\%/79.59\%; Sn 10.25\%/7.4\%; Pb 9.65\%/7.47\%; Fe 2.22/1.63\%; Ag 0.6\%/2.2\%.

From an archaeometric perspective, the data obtained in the current study by the combined EDXRF + Monte Carlo approach can, therefore, be considered in line with those obtained by other analytical approaches using, for example, the fundamental parameters approach $[35,36]$ in confirming ternary $\mathrm{Cu}-\mathrm{Sn} . \mathrm{Pb}$ alloys (with $\mathrm{Pb}$ and $\mathrm{Sn}$ in almost the same concentrations) as the ones commonly used in the Nuragic bronzes material culture. A compositional "outlier", though is represented by the Bultei sample where $\mathrm{Pb}>\mathrm{Sn}$.

The combined EDXRF/Monte Carlo protocol tested could be used as a fast tool and non-destructive tool in the routine analyses of the bulk composition of bronze artifacts stored in Museum collections aimed at assessing provenance of the raw materials and/or authenticity of archaeological and Cultural Heritage objects.

\section{Acknowledgements}

This work has been partially supported by L.R. 7 of Regione Sardegna Italy (project n. J71J12000530007).

\section{Appendix A. Supplementary data}

Supplementary data to this article can be found online at http://dx. doi.org/10.1016/j.sab.2016.07.011.

\section{References}

[1] R. Cesareo, A. Castellano, G. Buccolieri, S. Quarta, M. Marabelli, P. Santopadre, M. Leole, A. Brunetti, Portable equipment for energy dispersive X-ray fluorescence analysis of Giotto's frescoes in the Chapel of the Scrovegni, Nucl. Instrum. Methods Phys. Res., Sect. B 213 (2004) 703-706.

[2] M. Milazzo Radiation applications in art and archaeometry X-ray fluorescence applications to archaeometry. Possibility of obtaining non-destructive quantitative analyses, Nucl. Inst. Methods Phys. Res. B 213 (2004) 683-692

[3] R. Cesareo, A. Brunetti, S. Ridolfi, Pigment layers and precious metal sheets by energy-dispersive X-ray fluorescence analysis, X-Ray Spectrom. 37 (2008) 309-316.
[4] R. Cesareo, M.A. Rizzutto, A. Brunetti, D.V. Rao, Metal location and thickness in a multilayered sheet by measuring $\mathrm{K} \alpha / \mathrm{K} \beta, \mathrm{L} \alpha / \mathrm{L} \beta$ and $\mathrm{L} \alpha / \mathrm{L} \gamma$ X-ray ratios, Nucl. Instrum. Methods Phys. Res., Sect. B 267 (2009) 2890-2896.

[5] R. Cesareo, C. Calza, M. Dos Anjos, R.T. Lopes, A. Bustamante, S.J. Fabian, W. Alva, Z.L.R. Chero, Pre-Columbian alloys from the royal tombs of Sipan; energy dispersive X-ray fluorescence analysis with a portable equipment, Appl. Radiat. Isot. 68 (2010) 525-528.

[6] A. Guilherme, M. Manso, S. Pessanha, A. Zegzouti, M. Elaatmani, R. Bendaoud, J. Coroado, J.M.F. dos Santos, M.L. Carvalho, Micro-XRF for characterization of Moroccan glazed ceramics and Portuguese tiles, J. Instrum. 8 (2013) (art. C02055).

[7] R. Cesareo, A. Brunetti, R. D'Oriano, A. Canu, G.M. Demontis, A. Celauro, A Roman bronze statuette with gilded silver mask from Sardinia: an EDXRF study, Appl. Phys. A Mater. Sci. Process. 113 (2013) 905-910.

[8] N. Schiavon, A. Celauro, M. Manso, A. Brunetti, F. Susanna, Early Iron Age bronze statuettes in Southern Portugal: combining archaeological data with EDXRF and BSEM + EDS to assess provenance and production technology, Appl. Phys. A 113 (4) (2013) 865-875, http://dx.doi.org/10.1007/s00339-013-7747-7.

[9] N. Schiavon, V. Soria, A.M. Arruda, M. Beltrame, J. Mirão, "Losanga" decorated imitations of italic late republican black gloss tableware from South-Western Iberia: a multi-analytical/microchemical characterization, Microchem. J. 124 (2016) $712-718$.

[10] C. Bottaini, J. Mirão, M. Figuereido, A. Candeias, A. Brunetti, N. Schiavon, Energy dispersive X-ray fluorescence spectroscopy/Monte Carlo simulation approach for the non-destructive analysis of corrosion patina-bearing alloys in archaeological bronzes: the case of the bowl from the Fareleira 3 site (Vidigueira, South Portugal), Spectrochim. Acta B 103-104 (2015) 9-13.

[11] M. Manso, N. Schiavon, I. Queralt, A.M. Arruda, J.M. Sampaio, A. Brunetti, Alloy characterization of a 7th century BC archaeological bronze vase - overcoming patina constraints using Monte Carlo simulations, Spectrochim. Acta B 107 (2015) (2015) 93-96, http://dx.doi.org/10.1016/j.sab.2015.03.001.

[12] A. Brunetti, J. Fabian, C. Wester La Torre, N. Schiavon, A combined XRF/Monte Carlo Simulation study of multilayered Peruvian metal artefacts from the tomb of the priestess of Chornancap, Appl. Phys. A 122 (2016) 571, http://dx.doi.org/10.1007/ s00339-016-0096-6.

[13] A. Brunetti, A. DePalmas, F. di Gennaro, A. Serges, N. Schiavon, X-ray fluorescence spectroscopy (XRF) and Monte Carlo characterization of a unique nuragic artifact (Sardinia, Italy), Spectrochim. Acta B 121 (1) (2016) 18-21, http://dx.doi.org/10. 1016/j.sab.2016.04.007

[14] E. Jadot, N. Schiavon, M. Manso, The ceramics of Malpas of Zacapu, Michoacán, Mexico, during the Early and Middle Postclassic periods (900-1450 AD): micro-chemical characterization of surface paintings, Spectrochim. Acta B 119 (2016) 10-16, http:// dx.doi.org/10.1016/j.sab.2016.03.002.

[15] A.C. Mafalda, R.B. da Câmara, P. Strzelec, N. Schiavon, J. Mirão, A. Candeias, M.L Carvalho, M. Manso, White spots on smoke rings by Bruce Nauman: a case study on contemporary art conservation using microanalytical techniques, Microsc. Microanal. 21 (1) (2015) 15-19, http://dx.doi.org/10.1017/S1431927614013002.

[16] A. Celauro, N. Schiavon, A. Brunetti, L. Manfredi, F. Susanna, A. Dekayir, V. Graziani, D. Pargny, D. Ferro, Combining chemical data with GIS and PCA to investigate Phoenician-Punic Cu-metallurgy, Appl. Phys. A 114 (2014) 711-722, http://dx.doi. org/10.1007/s00339-013-8179-0.

[17] N. Schiavon, A. Candeias, T. Ferreira, M. da Conceicão Lopes, A. Carneiro, T. Calligaro, J. Mirão, A combined multi-analytical approach for the study of Roman glass from Southwest Iberia: synchrotron $\mu$-XRF, external-PIXE/PIGE and VP-BSEM-EDS, Archaeometry 54 (6) (2012) 974-996.

[18] R. Cesareo, A. Brunetti, Metal sheets thickness determined by energy-dispersive Xray fluorescence analysis, J. X-ray Sci. Technol. 16 (2008) 119-130.

[19] RT. Cesareo, JT. de Assis, C. Roldán, C. Bustamante, A. Brunetti, N. Schiavon, Multilayered samples reconstructed by measuring $\mathrm{Ka} / \mathrm{K} ß$ or $\mathrm{La} / \mathrm{L} ß \mathrm{X}$-ray intensity ratios by EDXRF, Nucl. Inst. Methods Phys. Res. B 312 (2013) 15-22.

[20] T. Trojek, Reconstruction of the relief of an investigated object with scanning X-ray fluorescence microanalysis and Monte Carlo simulations of surface effects, Appl. Radiat. Isot. 70 (2012) 1206-1209.

[21] P. Tack, M. Cotte, S. Bauters, E. Brun, D. Banerjee, W. Bras, C. Ferrero, D. Delattre, V. Mocella, L. Vincze, Tracking ink composition on Herculaneum papyrus scrolls: quantification and speciation of lead by X-ray based techniques and Monte Carlo simulations, Sci. Rep. 8 (2016) http://dx.doi.org/10.1038/srep20763 (Article number 20763)

[22] R.P. Gardner, J.M. Doster, Reduction of matrix effects in X-ray fluorescence analysis by the Monte Carlo, fundamental parameters method, Adv. X-ray Anal. 22 (1979) 343-356.

[23] R.P. Gardner, J.M. Doster, The complete spectral response for EDXRF systemscalculation by Monte Carlo and analysis applications. 1 Homogeneous samples, XRay Spectrom. 11 (1982) 173-18.

[24] R.P. Gardner, J.M. Doster, The complete spectral response for EDXRF systemscalculation by Monte Carlo and analysis applications. 1 Heterogeneous samples, X-Ray Spectrom. 11 (1982) 181-186.

[25] J.E. Fernandez, Monte Carlo computer simulation of the XRF intensity dependence on the propagation plane inclination. Comput. Phys. Commun. 54 (1989) 211-220.

[26] A. Depalmas, Le navicelle di bronzo della Sardegna nuragica, Ettore Gasperini Editore, Cagliari, 2005 (in Italian).

[27] U. Bottigli, A. Brunetti, B. Golosio, P. Oliva, S. Stumbo, L. Vincze, P. Randaccio, P. Bleuet, A. Simionovici, A. Somogyi, Voxel-based Monte Carlo simulation of X-ray imaging and spectroscopy experiments, Spectrochim. Acta B 59 (2004) 1747-1754.

[28] A. Brunetti, B. Golosio, A new Monte Carlo code for simulation of the effect of irregular surfaces on X-ray spectra, Spectrochim. Acta B 94-95 (2014) 58-62, http://dx.doi.org/10.1016/j.sab.2014.03.007. 
[29] L. Vincze, K. Janssen, F. Adams, A general Monte Carlo simulation of energy-dispersive X-ray fluorescence spectrometers-I: unpolarized radiation, homogeneous samples, X-Ray, X-Ray Spectrom. 48 (1993) 553-573.

[30] T. Schoonjans, L. Vincze, V.A. Solé, M. Sanchez del Rio, P. Brondeel, G. Silversmit, K. Appel, C. Ferrero, A general Monte Carlo simulation of energy-dispersive X-ray fluorescence spectrometers - part 5. Polarized radiation, stratified samples, cascade effects, M-lines, Spectrochim. Acta B 70 (2012) 10-23.

[31] B. Golosio, T. Schoonjans, A. Brunetti, P. Oliva, G.L. Masala, Monte Carlo simulation of $\mathrm{x}$-ray imaging and spectroscopy experiments using quadric geometry and variance reduction techniques, Comput. Phys. Commun. 185 (2014) 1044-1052.

[32] A. Brunetti, B. Golosio, T. Schoonjans, P. Oliva, Use of Monte Carlo simulations for cultural heritage X-ray fluorescence analysis, Spectrochim. Acta B 15-20 (2015).
[33] A. Brunetti, M. Sanchez del Rio, B. Golosio, A. Simionovici, A. Somogyi, A library for $\mathrm{X}$-ray-matter interaction cross sections for X-ray fluorescence applications, Spectrochim. Acta B 59 (2004) 1725-1731.

[34] T. Schoonjans, A. Brunetti, B. Golosio, M. Sanchez del Rio, V.A. Solé, C. Ferrero, L Vincze, The Xraylib library for X-ray-matter interactions. Recent developments, Spectrochim. Acta B 66 (2011) 776-784.

[35] J. Riederer, Metallanalysen sardischer Bronzen, Kunst und Kultur Sardiniens vom Neolithikum bis zum ende der Nuraghenzeit, Karlsruhe, 1980 156-160 (in German).

[36] F. Lo Schiavo, A. Giumlia-Mair, U. Sanna, R. Valera, Archaeometallurgy in Sardinia from the origins to the beginning of the Early Iron Age, Monographie Instrumentum, 30, 2005 (Montagnac). 